\title{
INCORPORATING EVIDENCE INTO CLINICAL GUIDELINES FOR THE MANAGEMENT OF DIABETES
}

Jeannine Liddle and Margaret Williamson

Centre for Clinical Policy and Practice

Public Health Division

NSW Health Department

D eveloping and implementing clinical guidelines for the management of diabetes are important components of the NSW Health Department's health outcomes approach to improving diabetes care ${ }^{1}$. The purpose of guidelines is to improve standards and outcomes of care by promoting interventions for which there is evidence of effectiveness and benefit. Where possible this evidence should be based on rigorous research.

This article outlines an explicit approach to identify, evaluate and incorporate scientific evidence into guidelines in a form that enables the quality of the evidence to be assessed. The approach comprises eight steps, summarised as a flow chart in Figure 3.

Evidence may be incorporated into clinical management guidelines either as an intrinsic part of guideline development or following the development of consensus guidelines. The method outlined here was developed using existing consensus guidelines.

\section{THE EIGHT STEPS}

\section{Background survey of the topic}

Reading general literature such as textbooks or overviews and consulting content experts provides important background information for developing the objective of the review of evidence. Important articles and keywords can be identified in this step and used when searching electronic databases.

Often the purpose for reviewing the evidence and the main issues are clear at the outset. If so, the process will begin at Step 2.

\section{Specification of the objective of the review of evidence}

Clear specification of the objective determines and limits the scope of the review of evidence. It thereby helps to ensure the volume of evidence is manageable and that irrelevant and unnecessary literature searches are avoided. Experts and intended guideline users may provide advice on what is important to know and what they would like to have clarified through the evidence.

Adverse consequences as well as benefits of an intervention are defined at this step. It is obviously important to identify adverse effects associated with any proposed recommendations in a guideline, and to modify the guidelines to prevent or minimise untoward effects. For example, in guidelines to improve blood glucose control in people with diabetes, a caution was included for older people who may experience unacceptable levels of hypoglycaemia with attempts to achieve optimal blood glucose control.

Exclusion criteria for studies should be considered at this step. Studies which do not include the guideline's target population or disease subgroup may be explicitly excluded.

\section{Specific literature searches}

The Cochrane Collaboration, an international network that systematically reviews the evidence for a wide range of interventions, should be contacted at an early stage to identify existing reviews or current reviews.
Evidence will be identified from electronic databases such as MEDLINE and CINAHL. A variety of search strategies should be used, including a focus on terms such as metaanalysis, randomised, clinical trial and keywords relating to the content area. Evidence can also be identified by content experts (especially on unpublished results or work in progress) and articles referred to in the sources mentioned or in other bibliographies. Negative studies - those which show non-significant effects - and studies showing adverse effects should be included.

This step may generate a large list of abstracts needing further classification in Step 4 before retrieving the full articles in Step 5 .

\section{Classification of the literature}

The literature identified in Step 3 is classified according to its general purpose and according to study type. Does the study measure the effects of interventions, or examine causes of a disease, or assess the accuracy of diagnostic tests?

The focus here should be on the methods section of the abstract rather than the results, because a knowledge of the results might bias the assessor in deciding whether to retrieve the full article in Step 5. The finding that no clinical studies are available in the topic under consideration should point to directions for future research.

\section{Retrieval of full articles}

This step entails deciding which articles to retrieve in full. It is important to record these decisions. Where many studies exist, only systematic reviews of randomised controlled trials (RCTs) or multicentre, randomised controlled trials should be pursued. Generally these studies provide the best evidence for assessing effects of interventions.

If no systematic reviews of RCTs or multicentre RCTs exist, a hierarchy of study types should be pursued, starting with single centre RCTs. If these are not available, observational studies should be assessed. Studies on adverse effects and studies done in Australia can be included as evidence even when they may not be as high in the hierarchy of study types as studies which show beneficial effects or studies done overseas. For example, evidence for beneficial effects of near-normal glycaemic control was derived from a systematic review of 16 randomised controlled trials ${ }^{2}$ and a large, multicentre $\mathrm{RCT}^{3}$ published after the systematic review. Australian information on hypoglycaemia as an adverse effect came from an observational study, as this was the best local evidence available.

\section{Assessment of the quality of the evidence}

This takes into account the extent to which systematic errors (bias) have been prevented in study design and execution. Bias may lead to an over- or under-estimation of the "true" effectiveness of an intervention.

A specially developed checklist was used for evaluating the evidence relating to the diabetes guidelines ${ }^{4}$. Other checklists are also available ${ }^{5}$.

7. Quantification of the strength of the evidence Where possible the strength of evidence should be quantified through meta-analysis techniques, summarising 


\section{FIGURE 3}

\section{METHOD FOR INCORPORATING EVIDENCE INTO GUIDELINES}

Start of process Disseminate and evaluate guidelines/ongoing review

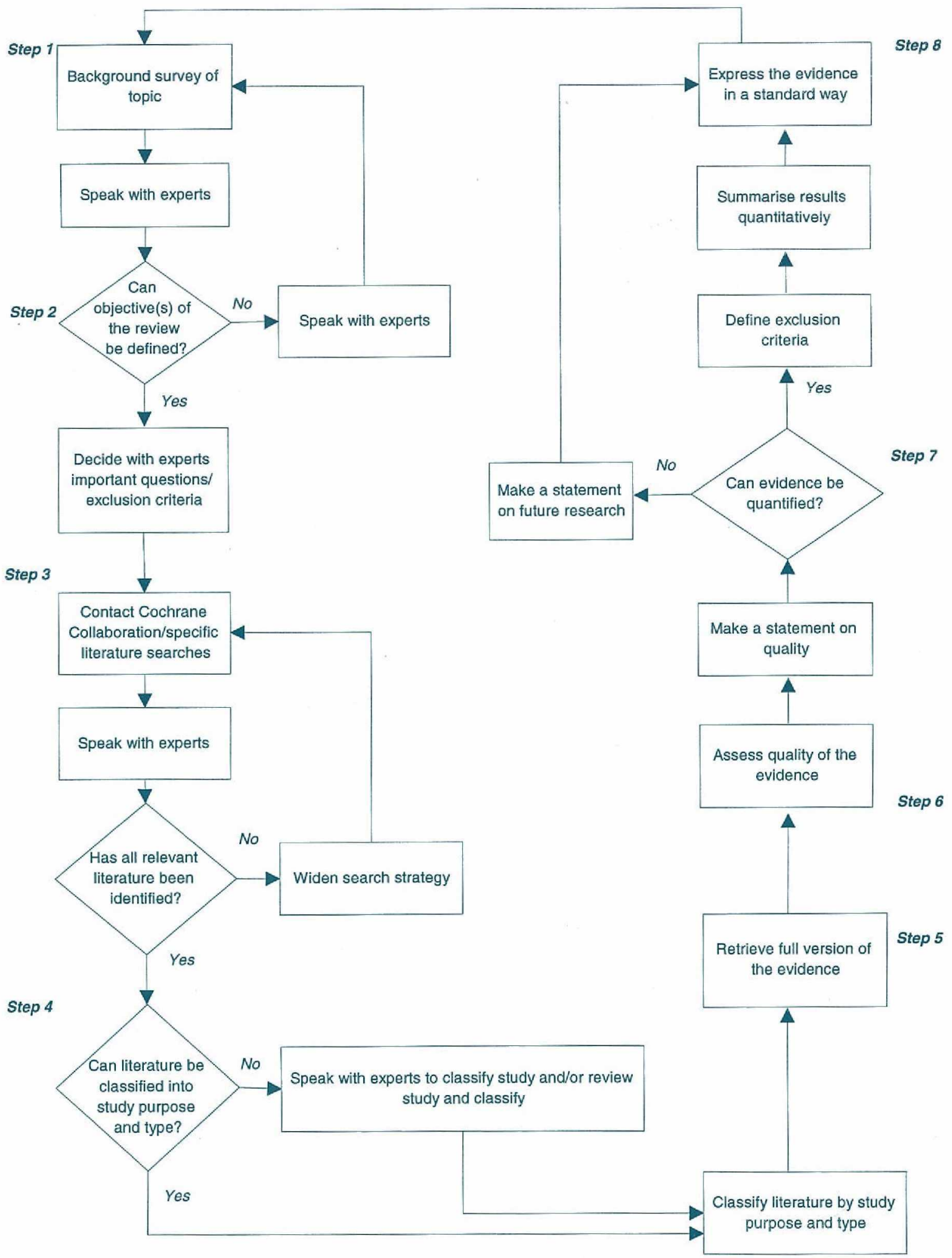




\section{Guidelines for diabetes management}

\section{$\checkmark$ Continued from page 145}

the results into a single point estimate with confidence intervals for both beneficial and adverse effects.

For example, the meta-analysis of $16 \mathrm{RCTs}^{2}$ on glycaemic control showed a significant reduction in progression of retinopathy with near normal glycaemic control over 2-5 years. This finding was summarised as an odds ratio of 0.49 with 95 per cent confidence interval of $0.28-0.85(\mathrm{p}=0.011)^{2}$.

Where results cannot be combined to quantify the strength of the evidence, the reason for this should be explained and the results of each study should be presented separately.

\section{Standardised expression of the evidence}

Evidence should be incorporated in guidelines in a standardised form. For example, the evidence for the diabetes guidelines includes:

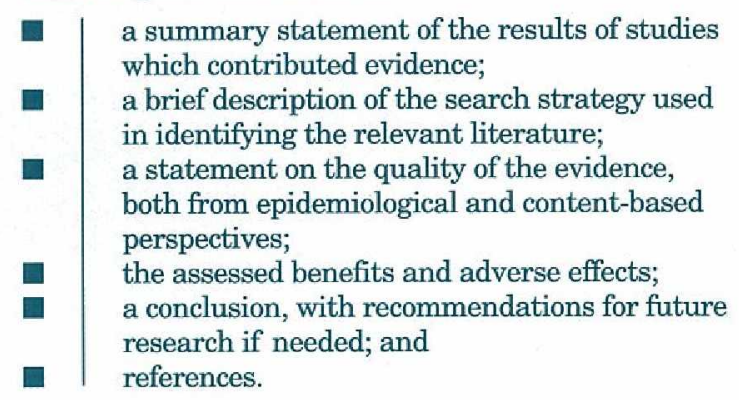

\section{CONCLUSION}

The eight steps described above make up a systematic method for incorporating evidence into guidelines for the clinical management of diabetes. This method can be applied to other content areas. By making the method explicit, the quality of evidence for diabetes guidelines can be assessed. For the guidelines to reflect current evidence, ongoing review is necessary.

\section{ACKNOWLEDGMENTS}

The contributions of Les Irwig, Lyn March, Anne Chamberlain, Stephen Colagiuri, Steven Boyages, Dennis Yue, Lesley Campbell, Robert Moses and Ruth Colagiuri are greatly appreciated.

\footnotetext{
1. Draft Interim Report-A health outcomes approach to diabetes. Chronic Diseases Unit, Public Health Division, NSW Health Department. Sydney, April 1995.

2. Wang P, Lau J, Chalmers T. Meta-analysis of the effects of intensive glycaemic control on late complications of type 1 diabetes mellitus. Online J Curr Clin Trials 1998; May 21; No 60.

3. The Diabetes Control and Complications Trial Research Group. The effect of intensive treatment of diabetes on the development and progression of long-term complications in insulin-dependent diabetes mellitus. New Engl J Med 1993; 329:977-86.

4. Irwig L, Liddle J, Williamson M. Evaluation checklist for evidencebased guidelines. NSW Health Department. Sydney, May 1995. 5. Moher D, Jadad A, Nichol G, Penman M, Tugwell P, Walsh S. Assessing the quality of randomised controlled trials: an annotated bibliography of scales and checklists. Controlled Clinical Trials 1995; $16: 62-73$
}

\section{WAITING LIST REDUCTION PROGRAM: RESULTS TO NOVEMBER 1995}

\author{
Nick Shiraev and Jean McGarry \\ Performance Management Division \\ NSW Health Department
}

The October and November 1995 issues of the

NSW Public Health Bulletin contained progress reports on the Waiting List Reduction Program ${ }^{12}$. This article describes the current status of the Program with results to the end of November 1995.

The Program was introduced to improve access to elective surgery in NSW public hospitals. The aim is to reduce the March 31, 1995 elective surgery waiting lists by 50 per cent within 12 months, concentrating on people who have been waiting more than six months for admission.

It has been a long-term practice of the NSW Health Department to categorise medical and surgical patients by clinical urgency. This helps to ensure they receive the best care in the most timely manner.

The clinical categories are:

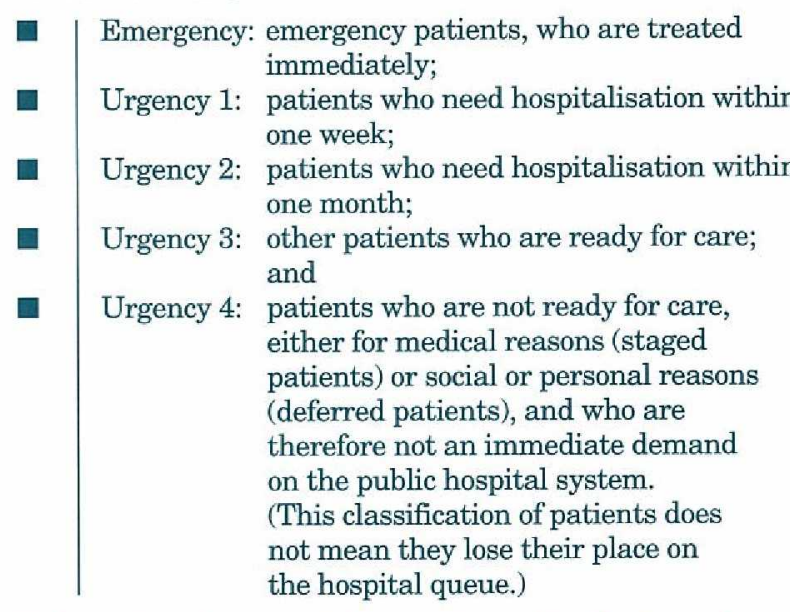

Unless otherwise stated, the results outlined below refer to Urgency 1,2 and 3 patients.

\section{RESULTS}

By November 30, 1995 the elective surgery waiting list had decreased from the March 31 figure of 44,707 to 24,701 patients (a reduction of 20,006 patients or 45 per cent).

Three Area and 10 District Health Services had achieved at least a 50 per cent reduction in the number of patients on elective surgery waiting lists (Table 3 ).

The vascular surgery list declined by 69 per cent. Urology declined by 55 per cent. Other notable reductions were obtained in ear, nose and throat surgery (down 41 per cent), general surgery (down 47 per cent), gynaecology (down 43 per cent), ophthalmology (down 46 per cent) and other surgery (down 75 per cent). Major decreases in list numbers were recorded for all clinical specialties. Expected waiting times were more than halved for ophthalmology and vascular surgery.

Particularly significant were reductions in numbers of people waiting longer than six months for procedures such 